\title{
THE IDEAL OF WEAKLY $p$-COMPACT OPERATORS AND ITS APPROXIMATION PROPERTY FOR BANACH SPACES
}

\author{
Ju Myung Kim \\ Sejong University, Department of Mathematics \\ Seoul 05006, Korea; kjm21@sejong.ac.kr
}

\begin{abstract}
We investigate the ideal $\mathcal{W}_{p}$ of weakly $p$-compact operators and its approximation property $\left(\mathcal{W}_{p}-\mathrm{AP}\right)$. We prove that

$$
\mathcal{W}_{p}=\mathcal{W}_{p} \circ \mathcal{W}_{p} \text { and } \mathcal{V}_{p}=\mathcal{K}_{u p} \circ \mathcal{W}_{p}^{-1}
$$

and that for $1<p \leq \infty$, a Banach space $X$ has the $\mathcal{W}_{p}$-AP if and only if the identity map on $X$ is approximated by finite rank operators on $X$ in the topology of uniform convergence on weakly $p$-compact sets. Also, we study the $\mathcal{W}_{p}$-AP for classical sequence spaces and dual spaces.
\end{abstract}

\section{Introduction}

The main subject of this paper originates from the classical approximation property (AP) and an operator ideal introduced by Sinha and Karn [SK1]. A Banach space $X$ is said to have the AP if

$$
\operatorname{id}_{X} \in \overline{\mathcal{F}(X, X)}^{\tau_{c}}
$$

where $\operatorname{id}_{X}$ is the identity map on $X, \mathcal{F}$ is the ideal of finite rank operators between Banach spaces and $\tau_{c}$ is the topology of uniformly compact convergence on the ideal $\mathcal{L}$ of all operators between Banach spaces. Grothendieck [G] systematically investigated the AP and one of the basic tools in $[\mathrm{G}]$ was a criterion of classical compactness such as the following.

A subset $K$ of a Banach space $X$ is relatively compact if and only if for every $\varepsilon>$ 0 , there exists $\left(x_{n}\right)_{n} \in c_{0}(X)$, the space of all null sequences in $X$, with $\left\|\left(x_{n}\right)_{n}\right\|_{\infty}:=$ $\sup _{n}\left\|x_{n}\right\| \leq \sup _{x \in K}\|x\|+\varepsilon$ such that

$$
K \subset\left\{\sum_{n=1}^{\infty} \alpha_{n} x_{n}:\left(\alpha_{n}\right)_{n} \in B_{\ell_{1}}\right\}
$$

where we denote by $B_{Z}$ the unit ball of a Banach space $Z$. It follows from this result that for every $T \in \mathcal{K}(Y, X)$, where $\mathcal{K}$ is the ideal of compact operators between Banach spaces,

(††) $\|T\|=\inf \left\{\left\|\left(x_{n}\right)_{n}\right\|_{\infty}:\left(x_{n}\right)_{n} \in c_{0}(X), T\left(B_{Y}\right) \subset\left\{\sum_{n=1}^{\infty} \alpha_{n} x_{n}:\left(\alpha_{n}\right)_{n} \in B_{\ell_{1}}\right\}\right\}$.

Sinha and Karn [SK1] was motivated by $(\dagger)$ to introduce a new compactness. Let $1 \leq p \leq \infty$. A subset $K$ of $X$ is said to be $p$-compact (respectively, weakly

https://doi.org/10.5186/aasfm.2020.4547

2010 Mathematics Subject Classification: Primary 46B28, 46B45, 47L20.

Key words: Weakly $p$-compact set, the ideal of weakly $p$-compact operators, the $\mathcal{W}_{p^{-}}$ approximation property. 
p-compact) if there exists $\left(x_{n}\right)_{n} \in \ell_{p}(X)$ (respectively, $\left.\ell_{p}^{w}(X)\right)$ such that

$$
K \subset p-c o\left(x_{n}\right)_{n}:=\left\{\sum_{n=1}^{\infty} \alpha_{n} x_{n}:\left(\alpha_{n}\right) \in B_{\ell_{p^{*}}}\right\},
$$

where $1 / p+1 / p^{*}=1$ and $\ell_{p}(X)$ is the Banach space with the norm $\|\cdot\|_{p}$ of all $X$-valued absolutely $p$-summable sequences (respectively, $\ell_{p}^{w}(X)$ is the Banach space with the norm $\|\cdot\|_{p}^{w}$ of all $X$-valued weakly $p$-summable sequences). When $p=\infty$, $\ell_{p}(X)$ (respectively, $\left.\ell_{p}^{w}(X)\right)$ is replaced by $c_{0}(X)$ (respectively, the space $c_{0}^{w}(X)$ of all weakly null sequences in $X)$. Also, when $p=1$, the unit ball $B_{\ell_{p^{*}}}$ is replaced by $B_{c_{0}}$. Note that every $p$-compact set is relatively compact and every weakly $p$-compact set $(1<p \leq \infty)$ is relatively weakly compact.

A linear map $T: Y \rightarrow X$ is $p$-compact (respectively, weakly p-compact) if $T\left(B_{Y}\right)$ is a $p$-compact (respectively, weakly $p$-compact) subset of $X$. The collection of all $p$-compact (respectively, weakly $p$-compact) operators from $Y$ to $X$ is denoted by $\mathcal{K}_{p}(Y, X)$ (respectively, $\left.\mathcal{W}_{p}(Y, X)\right)$. We remark that the notion of weakly $p$-compact set (the ideal of weakly $p$-compact operators) was already introduced and studied by Castillo and Sanchez as an another concept (see [CS, Definition 1.3]).

In view of $(\dagger \dagger)$, it is natural to consider the same way to measure $p$-compact operators. Delgado, Piñeiro and Serrano [DPS1] introduced an operator ideal norm on $\mathcal{K}_{p}$ in that way. For $T \in \mathcal{K}_{p}(Y, X)$, let

$$
\|T\|_{\mathcal{K}_{p}}:=\inf \left\{\left\|\left(x_{n}\right)_{n}\right\|_{p}:\left(x_{n}\right)_{n} \in \ell_{p}(X), T\left(B_{Y}\right) \subset p-c o\left(x_{n}\right)_{n}\right\} .
$$

Then $\left[\mathcal{K}_{p},\|\cdot\|_{\mathcal{K}_{p}}\right]$ is a Banach operator ideal [DPS1]. Ain, Lillemets and Oja [ALO] introduced and studied a more general form of the ideal $\left[\mathcal{K}_{p},\|\cdot\|_{\mathcal{K}_{p}}\right]$. We define a norm on $\mathcal{W}_{p}$ as in $\mathcal{K}_{p}$. For $T \in \mathcal{W}_{p}(Y, X)$, let

$$
\|T\|_{\mathcal{W}_{p}}:=\inf \left\{\left\|\left(x_{n}\right)_{n}\right\|_{p}^{w}:\left(x_{n}\right)_{n} \in \ell_{p}^{w}(X), T\left(B_{Y}\right) \subset p-c o\left(x_{n}\right)_{n}\right\} .
$$

Then $\left[\mathcal{W}_{p},\|\cdot\|_{\mathcal{W}_{p}}\right]$ is a Banach operator ideal (see Theorem 2.1).

Grothendieck $[\mathrm{G}]$ proved that a Banach space $X$ has the AP if and only if

$$
\mathcal{K}(Y, X)=\overline{\mathcal{F}(Y, X)} \|^{\|\cdot\|}
$$

for every Banach space $Y$, where the closure is in the operator norm topology. A more general notion extending this criterion was introduced by Lassalle and Turco [LT1], and Oja [O2]. For a Banach operator ideal $\left[\mathcal{A},\|\cdot\|_{\mathcal{A}}\right]$, a Banach space $X$ is said to have the $\mathcal{A}$-approximation property $\left(\mathcal{A}\right.$-AP) if $\mathcal{A}(Y, X)=\overline{\mathcal{F}(Y, X)}^{\|\cdot\|_{\mathcal{A}}}$ for every Banach space $Y$. Therefore a Banach space $X$ is said to have the $\mathcal{K}_{p}$-AP (respectively, $\left.\mathcal{W}_{p}-\mathrm{AP}\right)$ if

$$
\mathcal{K}_{p}(Y, X)=\overline{\mathcal{F}(Y, X)}{ }^{\|\cdot\| \mathcal{K}_{p}} \quad\left(\text { respectively, } \mathcal{W}_{p}(Y, X)=\overline{\mathcal{F}(Y, X)}{ }^{\|\cdot\| \mathcal{W}_{p}}\right)
$$

for every Banach space $Y$. The ideal $\mathcal{K}_{p}$, the $\mathcal{K}_{p}$-AP and their related subjects were investigated in [AO, ALO, CK, DP, DPS1, DPS2, DOPS, GLT, K1, K2, K3, K4, K6, K7, LT1, LT2, LT3, O2, P, PD, SK1, SK2] and so on. In this paper, we investigate the ideal $\mathcal{W}_{p}$ and the $\mathcal{W}_{p}$-AP as the following organization.

In Section 2, we prove that

$$
\mathcal{W}_{p}=\mathcal{W}_{p} \circ \mathcal{W}_{p} \quad \text { and } \quad \mathcal{V}_{p}=\mathcal{K}_{u p} \circ \mathcal{W}_{p}^{-1}
$$


In Section 3, we establish some characterizations of the $\mathcal{W}_{p}$-AP. Among them, for $1<p \leq \infty$, a Banach space $X$ has the $\mathcal{W}_{p}$-AP if and only if

$$
\operatorname{id}_{X} \in \overline{\mathcal{F}(X, X)}^{\tau_{w p}}
$$

where $\tau_{w p}$ is the topology of uniform convergence on weakly p-compact sets. In Section 4 , we check whether the classical sequence spaces have the $\mathcal{W}_{p}$-AP. As a consequence, it is shown that the $\mathrm{AP}$ does not imply the $\mathcal{W}_{p}$-AP and the $\mathcal{W}_{p}$-AP $(1<p<2)$ does not imply the AP in general. Also, we study the $\mathcal{W}_{p}$-AP for dual spaces. As a consequence, it is shown that for $1<p<\infty$, the dual space of a Banach space $X$ has the $\mathcal{W}_{p^{-}}$AP if and only if for every Banach space $Y, \mathcal{F}(X, Y)$ is dense in the space of quasi weakly $p$-nuclear operators from $X$ to $Y$.

\section{The ideal of weakly $p$-compact operators}

First, we need to show the following for the sake of the completeness of presentation.

Theorem 2.1. For every $1 \leq p \leq \infty,\left[\mathcal{W}_{p},\|\cdot\|_{\mathcal{W}_{p}}\right]$ is a Banach operator ideal.

Lemma 2.2. [K5, Corollary 3.6] Let $1 \leq p<\infty$ and let $T: X \rightarrow Y$ be a linear map.

(a) If $\left(y_{n}\right)_{n} \in \ell_{p}^{w}(Y)$, then $T\left(B_{X}\right) \subset p-c o\left(y_{n}\right)_{n}$ if and only if $\left\|T^{*} y^{*}\right\| \leq\left\|\left(y^{*}\left(y_{n}\right)\right)_{n}\right\|_{p}$ for every $y^{*} \in Y^{*}$.

(b) If $\left(y_{n}\right)_{n} \in c_{0}^{w}(Y)$, then $T\left(B_{X}\right) \subset \infty-\operatorname{co}\left(y_{n}\right)_{n}$ if and only if $\left\|T^{*} y^{*}\right\| \leq\left\|\left(y^{*}\left(y_{n}\right)\right)_{n}\right\|_{\infty}$ for every $y^{*} \in Y^{*}$.

Proof of Theorem 2.1. Let $X$ and $Y$ be Banach spaces. We only show the linearity of $\mathcal{W}_{p}(X, Y)$, the triangle inequality and completeness of $\|\cdot\|_{\mathcal{W}_{p}}$. The other conditions for an operator ideal are clear. Let $\left(T_{k}\right)_{k}$ be a sequence in $\mathcal{W}_{p}(X, Y)$ with $\sum_{k}\left\|T_{k}\right\|_{\mathcal{W}_{p}}<\infty$. Then $\sum_{k=1}^{\infty}\left\|T_{k}\right\|<\infty$ and so there exists a $T \in \mathcal{L}(X, Y)$ such that $\left\|\sum_{k=1}^{l} T_{k}-T\right\| \longrightarrow 0$ as $l \rightarrow \infty$.

Let $\varepsilon>0$ be given. For each $k \in \mathbf{N}$, let $\left(y_{n}^{k}\right)_{n} \in \ell_{p}^{w}(Y)$ be such that

$$
T_{k}\left(B_{X}\right) \subset p-c o\left(y_{n}^{k}\right)_{n} \quad \text { and } \quad\left\|\left(y_{n}^{k}\right)_{n}\right\|_{p}^{w} \leq\left\|T_{k}\right\|_{\mathcal{W}_{p}}+\frac{\varepsilon}{2^{k}}
$$

In the case $p=\infty$, let $\left(y_{n}^{k}\right)_{n} \in c_{0}^{w}(Y)$ and $\left\|\left(y_{n}^{k}\right)_{n}\right\|_{\infty} \leq\left\|T_{k}\right\|_{\mathcal{W}_{\infty}}+\varepsilon / 2^{k}$.

For each $k, n \in \mathbf{N}$, let

$$
z_{n}^{k}:=\frac{y_{n}^{k}}{\left(\left\|T_{k}\right\|_{\mathcal{W}_{p}}+\varepsilon / 2^{k}\right)^{1 / p^{*}}} \in Y
$$

In the case $p=\infty$, let

$$
z_{n}^{k}:=\frac{y_{n}^{k}}{\beta_{k}\left(\left\|T_{k}\right\|_{\mathcal{W}_{\infty}}+\varepsilon / 2^{k}\right)},
$$

where $\beta_{k}>1, \lim _{k \rightarrow \infty} \beta_{k}=\infty$ and

$$
\sum_{k=1}^{\infty} \beta_{k}\left(\left\|T_{k}\right\|_{\mathcal{W}_{\infty}}+\varepsilon / 2^{k}\right) \leq(1+\varepsilon) \sum_{k=1}^{\infty}\left(\left\|T_{k}\right\|_{\mathcal{W}_{\infty}}+\varepsilon / 2^{k}\right) .
$$


The sequence $\left(z_{m}\right)_{m}$ in $Y$ is defined as the following array:

$$
\begin{array}{cccccccc}
z_{1}^{1} & \rightarrow & z_{2}^{1} & & z_{3}^{1} & \cdots & z_{n}^{1} & \cdots \\
& & \downarrow & & \downarrow & & \downarrow & \\
z_{1}^{2} & \leftarrow & z_{2}^{2} & & z_{3}^{2} & \cdots & z_{n}^{2} & \cdots \\
& & & & \downarrow & & \downarrow & \\
z_{1}^{3} & \leftarrow & z_{2}^{3} & \leftarrow & z_{3}^{3} & \cdots & z_{n}^{3} & \cdots \\
& & & & & & \downarrow & \\
& & & \vdots & & \vdots & & \cdots \\
& & & & & & & \\
z_{1}^{n} & \leftarrow & z_{2}^{n} & \leftarrow & \cdots & \leftarrow & z_{n}^{n} & \cdots \\
& & & \vdots & & \vdots & & \vdots
\end{array}
$$

Then

$$
\sup _{y^{*} \in B_{Y^{*}}} \sum_{m=1}^{\infty}\left|y^{*}\left(z_{m}\right)\right|^{p} \leq \sum_{k=1}^{\infty} \frac{\left(\left\|\left(y_{n}^{k}\right)_{n}\right\|_{p}^{w}\right)^{p}}{\left(\left\|T_{k}\right\|_{\mathcal{W}_{p}}+\varepsilon / 2^{k}\right)^{p / p^{*}}} \leq \sum_{k=1}^{\infty}\left(\left\|T_{k}\right\|_{\mathcal{W}_{p}}+\frac{\varepsilon}{2^{k}}\right) .
$$

Thus

$$
\left\|\left(z_{m}\right)_{m}\right\|_{p}^{w} \leq\left(\sum_{k=1}^{\infty}\left\|T_{k}\right\|_{\mathcal{W}_{p}}+\varepsilon\right)^{1 / p}
$$

In the case $p=\infty$, we see that $\left(z_{m}\right)_{m} \in c_{0}^{w}(Y)$ and $\left\|\left(z_{m}\right)_{m}\right\|_{\infty} \leq 1$.

Now let $y^{*} \in Y^{*}$. Then, for each $k \in \mathbf{N}$, since $T_{k}\left(B_{X}\right) \subset p$-co $\left(y_{n}^{k}\right)_{n}$, by Lemma 2.2 $\left\|T_{k}^{*} y^{*}\right\| \leq\left\|\left(y^{*}\left(y_{n}^{k}\right)\right)_{n}\right\|_{p}$. Then we have

$$
\begin{aligned}
\left\|T^{*} y^{*}\right\| & \leq \sum_{k=1}^{\infty}\left\|T_{k}^{*} y^{*}\right\| \leq \sum_{k=1}^{\infty}\left\|\left(y^{*}\left(y_{n}^{k}\right)\right)_{n}\right\|_{p}=\sum_{k=1}^{\infty}\left(\left\|T_{k}\right\|_{\mathcal{W}_{p}}+\varepsilon / 2^{k}\right)^{1 / p^{*}}\left\|\left(y^{*}\left(z_{n}^{k}\right)\right)_{n}\right\|_{p} \\
& \leq\left(\sum_{k=1}^{\infty}\left\|T_{k}\right\|_{\mathcal{W}_{p}}+\varepsilon\right)^{1 / p^{*}}\left\|\left(y^{*}\left(z_{m}\right)\right)_{m}\right\|_{p} .
\end{aligned}
$$

In the case $p=\infty$, we see that

$$
\left\|T^{*} y^{*}\right\| \leq(1+\varepsilon)\left(\sum_{k=1}^{\infty}\left\|T_{k}\right\|_{\mathcal{W}_{\infty}}+\varepsilon\right)\left\|\left(y^{*}\left(z_{m}\right)\right)_{m}\right\|_{\infty} .
$$

For each $m \in \mathbf{N}$, let

$$
w_{m}:=\left(\sum_{k=1}^{\infty}\left\|T_{k}\right\|_{\mathcal{W}_{p}}+\varepsilon\right)^{1 / p^{*}} z_{m}
$$

In the case $p=\infty$, let

$$
w_{m}:=(1+\varepsilon)\left(\sum_{k=1}^{\infty}\left\|T_{k}\right\|_{\mathcal{W}_{\infty}}+\varepsilon\right) z_{m}
$$

Then since $\left(w_{m}\right)_{m} \in \ell_{p}^{w}(Y)$ or $\left(w_{m}\right)_{m} \in c_{0}^{w}(Y)$ for the case $p=\infty$, by Lemma 2.2,

$$
T\left(B_{X}\right) \subset p-c o\left(w_{m}\right)_{m} \text {. }
$$

Therefore $T \in \mathcal{W}_{p}(X, Y)$ and

$$
\|T\|_{\mathcal{W}_{p}} \leq\left\|\left(w_{m}\right)_{m}\right\|_{p}^{w} \leq(1+\varepsilon)\left(\sum_{k=1}^{\infty}\left\|T_{k}\right\|_{\mathcal{W}_{p}}+\varepsilon\right)
$$


Since $\varepsilon>0$ was arbitrary, $\|T\|_{\mathcal{W}_{p}} \leq \sum_{k=1}^{\infty}\left\|T_{k}\right\|_{\mathcal{W}_{p}}$.

The above proof can be applied to show that for every $l \in \mathbf{N}, \sum_{k>l} T_{k} \in$ $\mathcal{W}_{p}(X, Y)$ and $\left\|\sum_{k>l} T_{k}\right\|_{\mathcal{W}_{p}} \leq \sum_{k>l}\left\|T_{k}\right\|_{\mathcal{W}_{p}}$. Hence

$$
\left\|\sum_{k=1}^{l} T_{k}-T\right\|_{\mathcal{W}_{p}} \leq \sum_{k>l}\left\|T_{k}\right\|_{\mathcal{W}_{p}} \longrightarrow 0
$$

as $l \rightarrow \infty$.

Remark 2.3. Sinha and Karn [SK1, SK2] studied factorizations of $p$-compact and weakly $p$-compact operators, and defined the Banach operator ideal norms $\kappa_{p}(\cdot)$ and $\omega_{p}(\cdot)$, respectively, on $\mathcal{K}_{p}$ and $\mathcal{W}_{p}$, using those factorizations. Delgado, Piñeiro and Serrano [DPS1, Proposition 3.15] showed that $\kappa_{p}(\cdot)=\|\cdot\|_{\mathcal{K}_{p}}$. We can also show that $\omega_{p}(\cdot)=\|\cdot\|_{\mathcal{W}_{p}}$ using their proof.

Let $1 \leq p \leq \infty$ and let $X$ and $Y$ be Banach spaces. For $\hat{y}:=\left(y_{n}\right)_{n} \in \ell_{p}^{w}(Y)$ $\left(\left(y_{n}\right)_{n} \in c_{0}^{w}(Y)\right.$ when $\left.p=\infty\right)$, define the map $E_{\hat{y}}: \ell_{p^{*}} \rightarrow Y$ by

$$
E_{\hat{y}}\left(\alpha_{n}\right)_{n}=\sum_{n=1}^{\infty} \alpha_{n} y_{n} .
$$

Here $\ell_{p^{*}}$ is replaced by $c_{0}$ when $p=1$. For an operator $T: X \rightarrow Y$, the injective operator $T_{i n j}: X / \operatorname{ker}(T) \rightarrow Y$ is defined by

$$
T_{i n j}[x]=T x .
$$

The following result is essentially due to [SK1, Theorem 3.1].

Proposition 2.4. Let $1 \leq p<\infty$ and let $X$ and $Y$ be Banach spaces. Let $T: X \rightarrow Y$ be a linear map. Then $T \in \mathcal{W}_{p}(X, Y)$ if and only if there exist a quotient space $Z$ of $\ell_{p^{*}}\left(Z\right.$ is a quotient subspace of $c_{0}$ when $\left.p=1\right), R \in \mathcal{W}_{p}(X, Z)$ and injective $S \in \mathcal{W}_{p}(Z, Y)$ such that $T=S R$. In this case, $\|T\|_{\mathcal{W}_{p}}=\inf \|S\|_{\mathcal{W}_{p}}\|R\|_{\mathcal{W}_{p}}$, where the infimum is taken over all such factorizations.

Proof. The "if" part is clear and, in this case, $\|T\|_{\mathcal{W}_{p}} \leq \inf \|\cdot\| \mathcal{W}_{p}\|\cdot\| \mathcal{W}_{p}$.

Let $T \in \mathcal{W}_{p}(X, Y)$ and let $\varepsilon>0$ be given. Choose $\left(y_{n}\right)_{n} \in \ell_{p}^{w}(Y)$ such that $T\left(B_{X}\right) \subset p-c o\left(y_{n}\right)_{n}$ and $\left\|\left(y_{n}\right)_{n}\right\|_{p}^{w} \leq(1+\varepsilon)\|T\|_{\mathcal{W}_{p}}$. Then we see that the maps $E_{\hat{y}}: \ell_{p^{*}} \rightarrow Y$ and $\left(E_{\hat{y}}\right)_{i n j}: \ell_{p^{*}} / \operatorname{ker}\left(E_{\hat{y}}\right) \rightarrow Y$ are weakly $p$-compact and $\left\|\left(E_{\hat{y}}\right)_{i n j}\right\|_{\mathcal{W}_{p}} \leq$ $\left\|\left(y_{n}\right)_{n}\right\|_{p}^{w}$.

Now, for each $x \in X$, there exists $\left(\alpha_{n}\right)_{n} \in \ell_{p^{*}}$ such that $T x=\sum_{n=1}^{\infty} \alpha_{n} y_{n}$. Define the map

$$
\hat{T}: X \rightarrow \ell_{p^{*}} / \operatorname{ker}\left(E_{\hat{y}}\right) \quad \text { by } \hat{T} x=\left[\left(\alpha_{n}\right)_{n}\right] .
$$

Then $\hat{T}$ is well defined and linear, and we have the following commutative diagram.

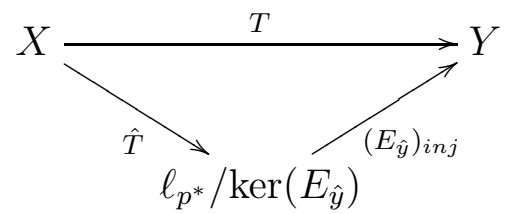

Consider the sequence $\left(\left[e_{n}\right]\right)_{n}$ in $\ell_{p^{*}} / \operatorname{ker}\left(E_{\hat{y}}\right)$, where each $e_{n}$ is the $n$-th standard unit vector in $\ell_{p^{*}}$. Then a simple verification shows that $\left(\left[e_{n}\right]\right)_{n} \in \ell_{p}^{w}\left(\ell_{p^{*}} / \operatorname{ker}\left(E_{\hat{y}}\right)\right)$ and

$$
\hat{T}\left(B_{X}\right) \subset p-c o\left(\left[e_{n}\right]\right)_{n} .
$$


Thus $\hat{T}$ is weakly $p$-compact and $\|\hat{T}\|_{\mathcal{W}_{p}} \leq 1$. Consequently,

$$
\inf \|\cdot\| \mathcal{W}_{p}\|\cdot\| \mathcal{W}_{p} \leq\left\|\left(y_{n}\right)_{n}\right\|_{p}^{w} \leq(1+\varepsilon)\|T\|_{\mathcal{W}_{p}} .
$$

Since $\varepsilon>0$ was arbitrary, we complete the proof.

Recall the composition operator ideal $\left[\mathcal{A} \circ \mathcal{B},\|\cdot\|_{\mathcal{A} \circ \mathcal{B}}\right]$ of operator ideals $\left[\mathcal{A},\|\cdot\|_{\mathcal{A}}\right]$ and $\left[\mathcal{B},\|\cdot\|_{\mathcal{B}}\right]$ (cf. [DF, Section 9.10]). Then by Proposition 2.4, we have:

Corollary 2.5. Let $1 \leq p<\infty$. Then $\left[\mathcal{W}_{p},\|\cdot\|_{\mathcal{W}_{p}}\right]=\left[\mathcal{W}_{p} \circ \mathcal{W}_{p},\|\cdot\|_{\mathcal{W}_{p} \circ \mathcal{W}_{p}}\right]$.

Remark 2.6. For the case $p=\infty$, the proof of Propsition 2.4 is invalid. Indeed, if the map

$$
\hat{T}: X \rightarrow \ell_{1} / \operatorname{ker}\left(E_{\hat{y}}\right)
$$

in the proof of Proposition 2.4 is a weakly $\infty$-compact operator, then it is a weakly compact operator. Hence the map $\hat{T}$ is a compact operator because $\ell_{1} / \operatorname{ker}\left(E_{\hat{y}}\right)$ has the Schur property (see, e.g., the proof of [JLO, Theorem 1.1]). Consequently, every weakly $\infty$-compact operator would be a compact operator. This is a contradiction.

We need a space of other vector valued sequences to introduce a stronger notion of the weakly $p$-compact operator. For $1 \leq p \leq \infty$, the closed subspace $\ell_{p}^{u}(X)$ of $\ell_{p}^{w}(X)$ consists of all sequences $\left(x_{n}\right)_{n}$ in a Banach space $X$ satisfying that

$$
\lim _{m \rightarrow \infty} \sup _{x^{*} \in B_{X^{*}}} \sum_{n \geq m}\left|x^{*}\left(x_{n}\right)\right|^{p}=0
$$

(cf. [DF, Section 8.2]). The sequence was called the unconditionally p-summable sequence in [K1]. Note that $\ell_{\infty}^{u}(X)=c_{0}(X)$. It is well known that for a sequence $\left(x_{n}\right)_{n}$ in $X,\left(x_{n}\right)_{n}$ is unconditionally 1-summable if and only if $\left(x_{n}\right)_{n}$ is unconditionally summable (cf. [DJT, Theorem 1.9]). The ideal $\left[\mathcal{K}_{u p},\|\cdot\|_{\mathcal{K}_{u p}}\right]$ of unconditionally $p$ compact operators was defined in [K1] by replacing $\ell_{p}(X)$ with $\ell_{p}^{u}(X)$ in the definition of the ideal of $p$-compact operators.

Let $\mathcal{V}$ be the ideal of completely continuous operators which take weakly null sequences to null sequences. For $1 \leq p<\infty$, let $\mathcal{V}_{p}$ be the ideal of operators which take weakly $p$-summable sequences to unconditionally $p$-summable sequences.

Recall that the right-hand quotient $\mathcal{A} \circ \mathcal{B}^{-1}$ of operator ideals $\mathcal{A}$ and $\mathcal{B}$ is the operator ideal that consists of all $T \in \mathcal{L}(X, Y)$ such that $T S \in \mathcal{A}(Z, Y)$ for every Banach space $Z$ and every $S \in \mathcal{B}(Z, X)$. It was shown in [JLO, Theorem 1.1] that

$$
\mathcal{V}=\mathcal{W}_{\infty} \circ \mathcal{W}^{-1}
$$

where $\mathcal{W}$ is the ideal of weakly compact operators.

Lemma 2.7. Let $1 \leq p \leq \infty$ and let $\left(x_{n}\right)_{n} \in \ell_{p}^{w}(X)\left(\left(x_{n}\right)_{n} \in c_{0}^{w}(X)\right.$ when $p=\infty)$. The operator $E_{\hat{x}}: \ell_{p^{*}} \rightarrow X$ is compact if and only if $\left(x_{n}\right)_{n} \in \ell_{p}^{u}(X)$.

Proof. We see that the adjoint operator $E_{\hat{x}}^{*}: X^{*} \rightarrow \ell_{p}\left(\ell_{p}\right.$ is replaced by $c_{0}$ when $p=\infty)$ is defined by

$$
E_{\hat{x}}^{*} x^{*}=\left(x^{*}\left(x_{n}\right)\right)_{n} .
$$

It is well known that the subset $\left\{\left(x^{*}\left(x_{n}\right)\right)_{n}: x^{*} \in B_{X^{*}}\right\}$ of $\ell_{p}$ is relatively compact if and only if $\left(x_{n}\right)_{n} \in \ell_{p}^{u}(X)$ (cf. [D, Exercises I.6 and II.6(i)]). Hence the conclusion follows.

Theorem 2.8. For $1 \leq p<\infty$,

$$
\mathcal{V}_{p}=\mathcal{K}_{u p} \circ \mathcal{W}_{p}^{-1}
$$


Proof. Let $X$ and $Y$ be Banach spaces. By definitions, it is clear that

$$
\mathcal{V}_{p}(X, Y) \subset \mathcal{K}_{u p} \circ \mathcal{W}_{p}^{-1}(X, Y) .
$$

In order to show the other part, let $T \in \mathcal{K}_{u p} \circ \mathcal{W}_{p}^{-1}(X, Y)$. Suppose that $T \notin$ $\mathcal{V}_{p}(X, Y)$. Then there exists $\left(x_{n}\right)_{n} \in \ell_{p}^{w}(X)$ such that $\left(T x_{n}\right)_{n} \notin \ell_{p}^{u}(Y)$.

Consider the following commutative diagram, where $q_{\hat{x}}: \ell_{p^{*}} \rightarrow \ell_{p^{*}} / \operatorname{ker}\left(E_{\hat{x}}\right)$ is the quotient operator.

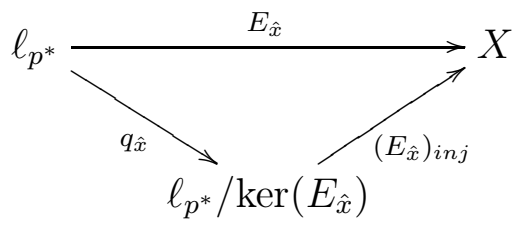

Note that $\left(E_{\hat{x}}\right)_{i n j}$ is a weakly $p$-compact operator. Since $T \in \mathcal{K}_{u p} \circ \mathcal{W}_{p}^{-1}(X, Y)$, $T\left(E_{\hat{x}}\right)_{i n j} \in \mathcal{K}_{u p}\left(\ell_{p^{*}} / \operatorname{ker}\left(E_{\hat{x}}\right), Y\right)$. Thus there exists $\left(y_{n}\right)_{n} \in \ell_{p}^{u}(Y)$ such that

$$
T\left(E_{\hat{x}}\right)_{i n j}\left(B_{\ell_{p^{*}} / \operatorname{ker}\left(E_{\hat{x}}\right)}\right) \subset p-c o\left(y_{n}\right)_{n} .
$$

Then we have

$$
E_{\widehat{T x}}\left(B_{\ell_{p^{*}}}\right)=T E_{\hat{x}}\left(B_{\ell_{p^{*}}}\right)=T\left(E_{\hat{x}}\right)_{i n j} q_{\hat{x}}\left(B_{\ell_{p^{*}}}\right) \subset p-c o\left(y_{n}\right)_{n} .
$$

Hence by Lemma 2.7, $\left(T x_{n}\right)_{n} \in \ell_{p}^{u}(Y)$. This is a contradiction.

Remark 2.9. The proof of Theorem 2.8 can be also applied to show that

$$
\mathcal{V}=\mathcal{K} \circ \mathcal{W}_{\infty}^{-1}
$$

\section{Characterizations of the $\mathcal{W}_{p}$-approximation property}

Let $1 \leq p \leq \infty$. For Banach spaces $X$ and $Y$, let $\tau_{w p}$ be the locally convex topology on $\mathcal{L}(X, Y)$ of uniform convergence on weakly p-compact sets, which is given by the seminorms

$$
p_{K}(T)=\sup _{x \in K}\|T x\|,
$$

where $K$ ranges over all weakly $p$-compact subsets of $X$. By definition of the weakly $p$-compact set, we see that the topology $\tau_{w p}$ is given by the seminorms

$$
p_{\hat{x}}(T)=\left\|\left(T x_{n}\right)_{n}\right\|_{p}^{w},
$$

where $\left(x_{n}\right)_{n} \in \ell_{p}^{w}(X)$. Then for a net $\left(T_{\alpha}\right)_{\alpha}$ in $\mathcal{L}(X, Y), \lim _{\alpha} T_{\alpha}=0$ in $\left(\mathcal{L}(X, Y), \tau_{w p}\right)$ if and only if

$$
\lim _{\alpha}\left\|\left(T_{\alpha} x_{n}\right)_{n}\right\|_{p}^{w}=0
$$

for every $\left(x_{n}\right)_{n} \in \ell_{p}^{w}(X)$.

First, we apply Proposition 2.4 to a characterization of the $\mathcal{W}_{p}$-AP.

Proposition 3.1. Let $1 \leq p<\infty$. A Banach space $X$ has the $\mathcal{W}_{p}-A P$ if and only if for every quotient space $Z$ of $\ell_{p^{*}}\left(Z\right.$ is a quotient space of $c_{0}$ when $\left.p=1\right)$ and every injective $R \in \mathcal{W}_{p}(Z, X)$,

$$
R \in \overline{\mathcal{F}(Z, X)}^{\tau_{w p}} .
$$


Proof. We only need to show the "if" part. Let $Y$ be a Banach space and let $T \in \mathcal{W}_{p}(Y, X)$. Let $\varepsilon>0$ be given. By Proposition 2.4, there exist a Banach space $W$, a quotient space $Z$ of $\ell_{p^{*}}, R_{1} \in \mathcal{W}_{p}(Y, W), R_{2} \in \mathcal{W}_{p}(W, Z)$ and injective $R \in \mathcal{W}_{p}(Z, X)$ such that the following diagram is commutative.

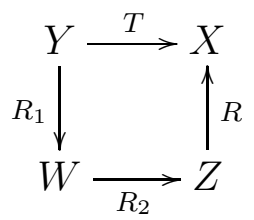

Then by our assumption, there exists an $S \in \mathcal{F}(Z, X)$ such that

$$
\varepsilon \geq\left\|R_{1}\right\|_{\mathcal{W}_{p}} \sup _{z \in R_{2}\left(B_{W}\right)}\|R z-S z\|=\left\|R_{1}\right\|_{\mathcal{W}_{p}}\left\|R R_{2}-S R_{2}\right\| \geq\left\|T-S R_{2} R_{1}\right\|_{\mathcal{W}_{p}} .
$$

Since $S R_{2} R_{1} \in \mathcal{F}(Y, X)$, we complete the proof.

We now obtain a similar characterization with the AP for the $\mathcal{W}_{p}$ - AP.

Theorem 3.2. Let $1<p<\infty$. The following statements are equivalent for a Banach space $X$.

(a) $X$ has the $\mathcal{W}_{p}-A P$.

(b) For every quotient space $Z$ of $\ell_{p^{*}}$ and every injective $R \in \mathcal{W}_{p}(Z, X), R \in$ $\overline{\mathcal{F}(Z, X)})^{\|\cdot\|}$.

(c) $\operatorname{id}_{X} \in \overline{\mathcal{F}(X, X)}^{\tau_{w p}}$.

(d) For every $\left(x_{n}\right)_{n} \in \ell_{p}^{w}(X), E_{\hat{x}} \in \overline{\left\{S E_{\hat{x}}: S \in \mathcal{F}(X, X)\right\}} \|^{\| \cdot \mathcal{W}_{p}}$.

(e) For every Banach space $Y$ and every $R \in \mathcal{W}_{p}(Y, X)$, $R \in \overline{\{S R: S \in \mathcal{F}(X, X)\}}^{\|\cdot\|_{\mathcal{w}_{p}}}$.

Proof. We show that (a) $\Rightarrow$ (b) $\Rightarrow$ (c) $\Rightarrow(\mathrm{d}) \Rightarrow$ (e) $\Rightarrow$ (a).

(a) $\Rightarrow$ (b) and (e) $\Rightarrow$ (a) are trivial.

(b) $\Rightarrow(\mathrm{c})$ : Let $\left(x_{n}\right)_{n} \in \ell_{p}^{w}(X)$ and let $\varepsilon>0$ be given. Consider the maps

$$
E_{\hat{x}}: \ell_{p^{*}} \rightarrow X \text { and }\left(E_{\hat{x}}\right)_{i n j}: \ell_{p^{*}} / \operatorname{ker}\left(E_{\hat{x}}\right) \rightarrow X \text {. }
$$

Then by (b), there exists an $S \in \mathcal{F}\left(\ell_{p^{*}} / \operatorname{ker}\left(E_{\hat{x}}\right), X\right)$ such that

$$
\left\|S-\left(E_{\hat{x}}\right)_{i n j}\right\| \leq \frac{\varepsilon}{2}
$$

We may write $S=\sum_{k=1}^{m} y_{k}^{*} \otimes x_{k}$, where $y_{k}^{*} \in\left(\ell_{p^{*}} / \operatorname{ker}\left(E_{\hat{x}}\right)\right)^{*}, x_{k} \in X$ for each $k=1, \ldots, m$ and $\sum_{k=1}^{m}\left\|x_{k}\right\|=1$. Since $\left(E_{\hat{x}}\right)_{i n j}$ is injective and $\ell_{p^{*}} / \operatorname{ker}\left(E_{\hat{x}}\right)$ is reflexive, $\left(E_{\hat{x}}\right)_{i n j}{ }^{* *}$ is injective. Thus $\left(\ell_{p^{*}} / \operatorname{ker}\left(E_{\hat{x}}\right)\right)^{*}=\overline{\left(E_{\hat{x}}\right)_{i n j}^{*}\left(X^{*}\right)}$. Thus for each $k=1, \ldots, m$, there exists an $x_{k}^{*} \in X^{*}$ such that

$$
\left\|y_{k}^{*}-\left(E_{\hat{x}}\right)_{i n j}^{*}\left(x_{k}^{*}\right)\right\| \leq \frac{\varepsilon}{2} \text {. }
$$

Consider the operator $\sum_{k=1}^{m} x_{k}^{*} \otimes x_{k} \in \mathcal{F}(X, X)$. Then for every $\left(\alpha_{n}\right) \in B_{\ell_{p^{*}}}$, we have

$$
\begin{aligned}
\left\|\sum_{k=1}^{m} x_{k}^{*}\left(\sum_{n=1}^{\infty} \alpha_{n} x_{n}\right) x_{k}-\sum_{n=1}^{\infty} \alpha_{n} x_{n}\right\| & =\left\|\sum_{k=1}^{m} x_{k}^{*}\left(E_{\hat{x}}\left(\alpha_{n}\right)_{n}\right) x_{k}-E_{\hat{x}}\left(\alpha_{n}\right)_{n}\right\| \\
& \left.=\| \sum_{k=1}^{m} x_{k}^{*}\left(\left(E_{\hat{x}}\right)_{i n j}\left[\left(\alpha_{n}\right)_{n}\right]\right) x_{k}-\left(E_{\hat{x}}\right)_{i n j}\left[\left(\alpha_{n}\right)_{n}\right]\right) \|
\end{aligned}
$$




$$
\begin{aligned}
= & \left.\| \sum_{k=1}^{m}\left(\left(E_{\hat{x}}\right)_{i n j}^{*} x_{k}^{*}\right)\left(\left[\left(\alpha_{n}\right)_{n}\right]\right) x_{k}-\left(E_{\hat{x}}\right)_{i n j}\left[\left(\alpha_{n}\right)_{n}\right]\right) \| \\
\leq & \left\|\sum_{k=1}^{m}\left(\left(E_{\hat{x}}\right)_{i n j}^{*} x_{k}^{*}\right)\left(\left[\left(\alpha_{n}\right)_{n}\right]\right) x_{k}-\sum_{k=1}^{m} y_{k}^{*}\left(\left[\left(\alpha_{n}\right)_{n}\right]\right) x_{k}\right\| \\
& \left.+\| \sum_{k=1}^{m} y_{k}^{*}\left(\left[\left(\alpha_{n}\right)_{n}\right]\right) x_{k}-\left(E_{\hat{x}}\right)_{i n j}\left[\left(\alpha_{n}\right)_{n}\right]\right) \| \leq \varepsilon .
\end{aligned}
$$

Hence $\operatorname{id}_{X} \in \overline{\mathcal{F}(X, X)}^{\tau_{w p}}$.

$(\mathrm{c}) \Rightarrow(\mathrm{d})$ : Let $\left(x_{n}\right) \in \ell_{p}^{w}(X)$ and let $\varepsilon>0$ be given. Then by (c), there exists an $S \in \mathcal{F}(X, X)$ such that

$$
\left\|\left(\left(S-\operatorname{id}_{X}\right) x_{n}\right)_{n}\right\|_{p}^{w} \leq \varepsilon .
$$

Since $\left(S E_{\hat{x}}-E_{\hat{x}}\right)\left(B_{\ell_{p^{*}}}\right)=p-c o\left(\left(S-\mathrm{id}_{X}\right) x_{n}\right)_{n}$ and $\left(\left(S-\mathrm{id}_{X}\right) x_{n}\right)_{n} \in \ell_{p}^{w}(X)$, we have

$$
\left\|S E_{\hat{x}}-E_{\hat{x}}\right\|_{\mathcal{W}_{p}} \leq\left\|\left(\left(S-\mathrm{id}_{X}\right) x_{n}\right)_{n}\right\|_{p}^{w} \leq \varepsilon
$$

$(\mathrm{d}) \Rightarrow(\mathrm{e})$ : Let $Y$ be a Banach space and let $R \in \mathcal{W}_{p}(Y, X)$. Let $\varepsilon>0$ be given. Then there exists $\left(x_{n}\right)_{n} \in \ell_{p}^{w}(X)$ such that $R\left(B_{Y}\right) \subset p$-co $\left(x_{n}\right)_{n}$. By $(\mathrm{d})$, there exists an $S \in \mathcal{F}(X, X)$ such that

$$
\left\|S E_{\hat{x}}-E_{\hat{x}}\right\|_{\mathcal{W}_{p}} \leq \varepsilon / 2 .
$$

Now, let $\left(z_{n}\right)_{n} \in \ell_{p}^{w}(X)$ be such that $\left(S E_{\hat{x}}-E_{\hat{x}}\right)\left(B_{\ell_{p^{*}}}\right) \subset p$-co $\left(z_{n}\right)_{n}$ and $\left\|\left(z_{n}\right)_{n}\right\|_{p}^{w} \leq$ $\left\|S E_{\hat{x}}-E_{\hat{x}}\right\|_{\mathcal{W}_{p}}+\varepsilon / 2$. Since $(S R-R)\left(B_{Y}\right) \subset p-c o\left(\left(S-\operatorname{id}_{X}\right) x_{n}\right)_{n}=\left(S E_{\hat{x}}-E_{\hat{x}}\right)\left(B_{\ell_{p^{*}}}\right)$, we have

$$
\|S R-R\|_{\mathcal{W}_{p}} \leq\left\|\left(z_{n}\right)_{n}\right\|_{p}^{w} \leq\left\|S E_{\hat{x}}-E_{\hat{x}}\right\|_{\mathcal{W}_{p}}+\varepsilon / 2 \leq \varepsilon .
$$

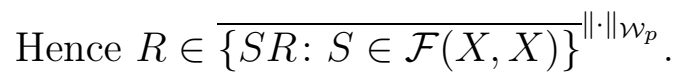

Remark 3.3. In Theorem 3.2, (c), (d) and (e) are also equivalent for the case $p=1$ and $p=\infty$.

Lima, Nygaard, and Oja [LNO] proved that if $K$ is a balanced convex and weakly compact set in the unit ball $B_{X}$ of a Banach space $X$, then there exists a linear subspace $Z$ of $X$, equipped with a different norm which makes it a reflexive Banach space, such that the formal identity map $J_{Z}: Z \longrightarrow X$ is a weakly compact operator and $K \subset B_{Z} \subset B_{X}$. Moreover, Oja showed

Lemma 3.4. [O1, Corollary 4.3]

$$
\mathcal{F}(Z, X) \subset \overline{\left\{S J_{Z}: S \in \mathcal{F}(X, X)\right\}} \|^{\|\cdot\|} .
$$
$\mathcal{L}$.

We denote by $\tau_{w c}$ the topology of uniform convergence on weakly compact sets on

Theorem 3.5. The following statements are equivalent for a Banach space $X$.

(a) $X$ has the $\mathcal{W}_{\infty}-A P$.

(b) $X$ has the AP and Schur's property.

(c) $X$ has the $\mathcal{W}$-AP.

(d) $\operatorname{id}_{X} \in \overline{\mathcal{F}(X, X)}^{\tau_{w c}}$.

(e) $\operatorname{id}_{X} \in \overline{\mathcal{F}(X, X)}^{\tau_{w}}$. 
Proof. We show that (a) $\Rightarrow$ (b) $\Rightarrow$ (c) $\Rightarrow$ (d) $\Rightarrow$ (e) $\Rightarrow$ (a). (d) $\Rightarrow$ (e) is trivial and (e) $\Rightarrow$ (a) follows from Remark 3.3. (a) $\Rightarrow$ (b): If $X$ has the $\mathcal{W}_{\infty}$-AP, then clearly it has the AP.

A Banach space $Z$ has the Schur property if (and only if)

$$
\mathcal{W}_{\infty}(Y, Z) \subset \mathcal{K}(Y, Z)
$$

for every Banach space $Y$. Indeed, if $\left(z_{n}\right)_{n} \in c_{0}^{w}(Z)$, then the operator

$$
E_{\hat{z}} \in \mathcal{W}_{\infty}\left(\ell_{1}, Z\right)
$$

If $\mathcal{W}_{\infty}\left(\ell_{1}, Z\right) \subset \mathcal{K}\left(\ell_{1}, Z\right)$, then we see that $\left\{z_{n}\right\}_{n=1}^{\infty}$ is a relatively compact subset of $Z$. Therefore $\left(z_{n}\right)_{n} \in c_{0}(Z)$.

By (a), $X$ has the Schur property.

(b) $\Rightarrow(\mathrm{c})$ : By (b), for every Banach space $Y$,

$$
\mathcal{W}(Y, X)=\mathcal{K}(Y, X)=\overline{\mathcal{F}(Y, X)}{ }^{\|\cdot\|} .
$$

(c) $\Rightarrow(\mathrm{d})$ : Let $K$ be a weakly compact subset of $X$ and let $\varepsilon>0$ be given. We may assume that $K$ is a balanced convex and weakly compact subset of $B_{X}$. Then by (c) and Lemma 3.4,

$$
J_{Z} \in \overline{\mathcal{F}(Z, X)}^{\|\cdot\|}={\overline{\left\{S J_{Z}: S \in \mathcal{F}(X, X)\right\}}}^{\|\cdot\|} .
$$

Hence there exists an $S \in \mathcal{F}(X, X)$ such that

$$
\varepsilon \geq\left\|J_{Z}-S J_{Z}\right\| \geq \sup _{x \in K}\left\|J_{Z} x-S J_{Z} x\right\|=\sup _{x \in K}\|x-S x\| .
$$

In the present paper, we do not know whether the $\mathcal{W}_{1}$-AP for a Banach space $X$ is equivalent to that $\operatorname{id}_{X} \in \overline{\mathcal{F}(X, X)}^{\tau_{w 1}}$.

\section{The $\mathcal{W}_{p}$-approximation property for the spaces $\ell_{p}, c_{0}, \ell_{\infty}$, and dual spaces}

In [K2], the $\mathcal{K}_{u p}$-AP was investigated and it was shown that if a Banach space $X$ has the AP, then $X$ has the $\mathcal{K}_{u p}$-AP for every $1 \leq p<\infty$.

Example 4.1. (The $\mathcal{W}_{1}$-AP) It follows from a result of Bessaga and Pelczyński that a Banach space $X$ does not contain an isomorphic copy of $c_{0}$ if and only if $\ell_{1}^{w}(X)=\ell_{1}^{u}(X)$ (cf. [M, Theorem 4.3.12]). Then for those Banach spaces $X, \mathcal{W}_{1}(Y, X)$ is isometrically equal to $\mathcal{K}_{u 1}(Y, X)$ for every Banach space $Y$. Consequently, for $1 \leq p<\infty$, since $\ell_{p}$ has the AP, it has the $\mathcal{W}_{1}$-AP.

On the other hand, $\mathcal{W}_{1}\left(c_{0}, X\right)=\mathcal{L}\left(c_{0}, X\right)$ for every Banach space $X$. Indeed, if $T \in \mathcal{L}\left(c_{0}, X\right)$, then

$$
T=\sum_{n=1}^{\infty} e_{n}^{*} \otimes T e_{n},
$$

where each $e_{n}$ and $e_{n}^{*}$, respectively, are the standard unit vectors in $c_{0}$ and $\ell_{1}$, respectively. Since $\left(T e_{n}\right)_{n} \in \ell_{1}^{w}(X)$, we see that $T \in \mathcal{W}_{1}\left(c_{0}, X\right)$. Then $c_{0}$ and $\ell_{\infty}$ do not have the $\mathcal{W}_{1}$-AP because the inclusion map from $c_{0}$ to $c_{0}$ (respectively, $\ell_{\infty}$ ) is not compact.

Example 4.2. (The $\mathcal{W}_{p}$-AP $(1<p<\infty)$ ) Let $1<p<\infty$ be fixed. It is known that $1 \leq q<p^{*}$ if and only if $\ell_{p}^{w}\left(\ell_{q}\right)=\ell_{p}^{u}\left(\ell_{q}\right)$ (cf. [DF, Ex. 8.4(b)]). Thus for $1 \leq q<p^{*}, \mathcal{W}_{p}\left(Y, \ell_{q}\right)$ is isometrically equal to $\mathcal{K}_{u p}\left(Y, \ell_{q}\right)$ for every Banach space $Y$. It follows that $\ell_{q}$ has the $\mathcal{W}_{p}$-AP for $1 \leq q<p^{*}$. 
On the other hand, as in Example 4.1, we see that $\mathcal{W}_{p}\left(\ell_{p^{*}}, X\right)=\mathcal{L}\left(\ell_{p^{*}}, X\right)$ for every Banach space $X$. Then $\ell_{q}\left(q \geq p^{*}\right), c_{0}$ and $\ell_{\infty}$ do not have the $\mathcal{W}_{p}$-AP because the inclusion map from $\ell_{p^{*}}$ to $\ell_{q}$ (respectively, $c_{0}$ and $\ell_{\infty}$ ) is not compact.

Example 4.3. (The $\mathcal{W}_{\infty}$-AP) From Theorem $3.5(\mathrm{a}) \Leftrightarrow(\mathrm{b}), \ell_{1}$ has the $\mathcal{W}_{\infty}$-AP but $\ell_{p}(1<p<\infty), c_{0}$ and $\ell_{\infty}$ do not have the $\mathcal{W}_{\infty}$-AP.

Example 4.4. In view of the above examples, the AP does not imply the $\mathcal{W}_{p^{-}}$ AP in general. Now, let $1<p<2$ be fixed and let $S_{p}$ be Szankowski's subspace [S] of $\ell_{p}$, which fails to have the AP. Since $1<p<2<p^{*}, \ell_{p}^{w}\left(\ell_{p}\right)=\ell_{p}^{u}\left(\ell_{p}\right)$. In particular, $\ell_{p}^{w}\left(S_{p}\right)=\ell_{p}^{u}\left(S_{p}\right)$. Thus $\mathcal{W}_{p}\left(Y, S_{p}\right)$ is isometrically equal to $\mathcal{K}_{u p}\left(Y, S_{p}\right)$ for every Banach space $Y$. In [K4, Section 5], it was observed that $S_{p}$ has the $\mathcal{K}_{u p}$-AP, hence it has the $\mathcal{W}_{p}$-AP. Also, the $\mathcal{W}_{\infty}$-AP implies the AP. In the present paper, for $p=1$ or $2 \leq p<\infty$, we do not know whether the $\mathcal{W}_{p}$-AP implies the AP.

We now consider the $\mathcal{W}_{p}$-AP for dual spaces. In [K5], a weaker notion of the $p$ nuclear operator was introduced (see, e.g., [DJT, p. 111] for the $p$-nuclear operator). For $1 \leq p \leq \infty$, we say that an operator $T: X \rightarrow Y$ is weakly $p$-nuclear if it is represented as

$$
T=\sum_{n=1}^{\infty} x_{n}^{*} \otimes y_{n}
$$

where $\left(x_{n}^{*}\right)_{n} \in \ell_{p}^{w}\left(X^{*}\right)\left(\left(x_{n}^{*}\right)_{n} \in c_{0}^{w^{*}}\left(X^{*}\right)\right.$ when $\left.p=\infty\right)$ and $\left(y_{n}\right)_{n} \in \ell_{p^{*}}^{w}(Y)\left(\left(y_{n}\right)_{n} \in\right.$ $c_{0}^{w}(Y)$ when $\left.p=1\right)$. Here $c_{0}^{w^{*}}\left(X^{*}\right)$ is the space of all weak* null sequences in $X^{*}$. We denote the space of all weakly $p$-nuclear operators from $X$ to $Y$ by $\mathcal{N}_{w p}(X, Y)$ and define a norm on $\mathcal{N}_{w p}(X, Y)$ by

$$
\|T\|_{\mathcal{N}_{w p}}:=\inf \left\|\left(x_{n}^{*}\right)_{n}\right\|_{p}^{w}\left\|\left(y_{n}\right)_{n}\right\|_{p^{*}}^{w}
$$

where the infimum is taken over all such weakly $p$-nuclear representations of $T$. Then $\left[\mathcal{N}_{w p},\|\cdot\|_{\mathcal{N}_{w p}}\right]$ is a Banach operator ideal [K5, Theorem 2.1]. It was shown in [K5, Theorem 3.2] that $\left[\mathcal{W}_{p},\|\cdot\|_{\mathcal{W}_{p}}\right]$ is equal to the surjective hull of $\left[\mathcal{N}_{w p^{*}},\|\cdot\|_{\mathcal{N}_{w p^{*}}}\right]$.

In [K5], a weaker notion of the quasi $p$-nuclear operator of Persson and Pietsch $[\mathrm{PP}]$ was introduced. For $1 \leq p \leq \infty$, a linear map $T: X \rightarrow Y$ is called quasi weakly $p$-nuclear if there exists $\left(x_{n}^{*}\right)_{n} \in \ell_{p}^{w}\left(X^{*}\right)\left(\left(x_{n}^{*}\right)_{n} \in c_{0}^{w^{*}}\left(X^{*}\right)\right.$ when $\left.p=\infty\right)$ such that

$$
\|T x\| \leq\left\|\left(x_{n}^{*}(x)\right)_{n}\right\|_{p}
$$

for every $x \in X$. We denote the space of all quasi weakly $p$-nuclear operators from $X$ to $Y$ by $\mathcal{N}_{w p}^{Q}(X, Y)$. For $T \in \mathcal{N}_{w p}^{Q}(X, Y)$, let $\|T\|_{\mathcal{N}_{w p}^{Q}}:=\inf \left\|\left(x_{n}^{*}\right)_{n}\right\|_{p}^{w}$, where the infimum is taken over all such inequalities. It was shown in [K5, Theorem 3.3] that $\left[\mathcal{N}_{w p}^{Q},\|\cdot\|_{\mathcal{N}_{w p}^{Q}}\right]$ is equal to the injective hull of $\left[\mathcal{N}_{w p},\|\cdot\|_{\mathcal{N}_{w p}}\right]$.

Let $\left[\mathcal{A},\|\cdot\|_{\mathcal{A}}\right]^{\text {dual }}$ be the dual ideal of a Banach operator ideal $\left[\mathcal{A},\|\cdot\|_{\mathcal{A}}\right]$ (cf. [DF, Section 9.9]).

Lemma 4.5 (K6, Proposition 4.9). Let $\mathcal{A}$ and $\mathcal{B}$ be Banach operator ideals. If $\mathcal{A} \subset \mathcal{B}^{\text {dual }}$ and $\mathcal{B} \subset \mathcal{A}^{\text {dual }}$, then the dual space of a Banach space $X$ has the $\mathcal{A}$-AP if and only if for every Banach space $Y, \mathcal{B}\left(X, Y^{*}\right)=\overline{\mathcal{F}\left(X, Y^{*}\right)}\left\|^{\cdot}\right\|_{\mathcal{B}}$.

In [K5, Theorem 3.7], it was shown that for $1 \leq p<\infty, \mathcal{W}_{p} \subset\left(\mathcal{N}_{w p}^{Q}\right)$ dual and $\mathcal{N}_{w p}^{Q} \subset \mathcal{W}_{p}^{\text {dual }}$. From Lemma 4.5, we have: 
Corollary 4.6. Let $1 \leq p<\infty$. The dual space of a Banach space $X$ has the $\mathcal{W}_{p}-A P$ (respectively, $\mathcal{N}_{w p}^{Q}-A P$ ) if and only if for every Banach space $Y, \mathcal{N}_{w p}^{Q}\left(X, Y^{*}\right)=$ $\overline{\mathcal{F}\left(X, Y^{*}\right)}\|\cdot\|_{\mathcal{N}_{w p}^{Q}}$ (respectively, $\left.\mathcal{W}_{p}\left(X, Y^{*}\right)=\overline{\mathcal{F}\left(X, Y^{*}\right)}\|\cdot\|_{\mathcal{W}_{p}}\right)$.

Proposition 4.7. Let $1 \leq p \leq \infty$ and let $X$ and $Y$ be Banach spaces. Let $T: X \rightarrow Y$ be a linear map. Then $T \in \mathcal{N}_{w p}^{Q}(X, Y)$ if and only if there exist a closed subspace $Z$ of $\ell_{p}\left(Z\right.$ is a closed subspace of $c_{0}$ when $\left.p=\infty\right), R \in \mathcal{N}_{w p}^{Q}(X, Z)$ and $S \in \mathcal{N}_{w p}^{Q}(Z, Y)$ such that $T=S R$. In this case, $\|T\|_{\mathcal{N}_{w p}^{Q}}=\inf \|S\|_{\mathcal{N}_{w p}^{Q}}\|R\|_{\mathcal{N}_{w p}^{Q}}$, where the infimum is taken over all such factorizations.

Proof. The "if" part is clear and, in this case, $\|T\|_{\mathcal{N}_{w p}^{Q}} \leq \inf \|\cdot\|_{\mathcal{N}_{w p}^{Q}}\|\cdot\|_{\mathcal{N}_{w p}^{Q}}$. that

Let $T \in \mathcal{N}_{w p}^{Q}(X, Y)$. Let $\varepsilon>0$ be given. Then there exists $\left(x_{n}^{*}\right)_{n} \in \ell_{p}^{w}\left(X^{*}\right)$ such

$$
\|T x\| \leq\left\|\left(x_{n}^{*}(x)\right)_{n}\right\|_{p}
$$

for every $x \in X$ and $\left\|\left(x_{n}^{*}\right)_{n}\right\|_{p}^{w} \leq\|T\|_{\mathcal{N}_{w p}^{Q}}+\varepsilon$. Consider the linear subspace

$$
Z_{0}=\left\{\left(x_{n}^{*}(x)\right)_{n}: x \in X\right\}
$$

of $\ell_{p}\left(\ell_{p}\right.$ is replaced by $c_{0}$ when $\left.p=\infty\right)$ and the map

$$
J_{0}: Z_{0} \rightarrow Y, \quad J_{0}\left(x_{n}^{*}(x)\right)_{n} \mapsto T x .
$$

Then it follows that $J_{0}$ is well defined and linear, and $\left\|J_{0}\right\| \leq 1$. Let $J: Z:=\overline{Z_{0}} \rightarrow Y$ be the linear continuous extension of $J_{0}$. Define the operator $U: X \rightarrow Z$ by

$$
U x=\left(x_{n}^{*}(x)\right)_{n} .
$$

Then $U$ is quasi weakly $p$-nuclear and $\|U\|_{\mathcal{N}_{w p}^{Q}} \leq\|T\|_{\mathcal{N}_{w p}^{Q}}+\varepsilon$.

Now, we obtain the following commutative diagram:

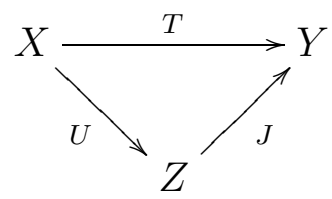

Consider the sequence $\left(e_{n}^{*}\right)_{n}$ of standard unit vectors in $\ell_{p^{*}}$. Then $\left(\left.e_{n}^{*}\right|_{Z}\right)_{n} \in \ell_{p}^{w}\left(Z^{*}\right)$ and $\left\|\left(\left.e_{n}^{*}\right|_{Z}\right)_{n}\right\|_{p}^{w} \leq 1$. Since for every $x \in X$,

$$
\left\|J\left(x_{k}^{*}(x)\right)_{k}\right\|_{Y}=\|T x\|_{Y} \leq\left\|\left(x_{k}^{*}(x)\right)_{k}\right\|_{p}=\left\|\left(\left\langle\left. e_{n}^{*}\right|_{Z},\left(x_{k}^{*}(x)\right)_{k}\right\rangle\right)_{n}\right\|_{p},
$$

we can check that $\|J z\|_{Y} \leq\left\|\left(\left\langle\left. e_{n}^{*}\right|_{Z}, z\right\rangle\right)_{n}\right\|_{p}$ for every $z \in Z$. Hence $J$ is quasi weakly $p$-nuclear and $\|J\|_{\mathcal{N}_{w p}^{Q}} \leq 1$, and inf $\|\cdot\|_{\mathcal{N}_{w p}^{Q}}\|\cdot\|_{\mathcal{N}_{w p}^{Q}} \leq\|T\|_{\mathcal{N}_{w p}^{Q}}+\varepsilon$.

Corollary 4.8. Let $1 \leq p \leq \infty$. Then $\left[\mathcal{N}_{w p}^{Q},\|\cdot\|_{\mathcal{N}_{w p}^{Q}}\right]=\left[\mathcal{N}_{w p}^{Q} \circ \mathcal{N}_{w p}^{Q},\|\cdot\|_{\mathcal{N}_{w p}^{Q} \circ \mathcal{N}_{w p}^{Q}}\right]$.

Proposition 4.9. Let $1 \leq p \leq \infty$. A Banach space $X$ has the $\mathcal{N}_{w p}^{Q}-A P$ if (and only if) for every closed subspace $Y$ of $\ell_{p}\left(\ell_{p}\right.$ is replaced by $c_{0}$ when $\left.p=\infty\right)$, $\mathcal{N}_{w p}^{Q}(Y, X) \subset \overline{\mathcal{F}(Y, X)}\|\cdot\|$

Proof. Let $Y$ be a Banach space and let $T \in \mathcal{N}_{w p}^{Q}(Y, X)$. Let $\varepsilon>0$ be given. By Proposition 4.7, there exists a closed subspace $Z$ of $\ell_{p}, R \in \mathcal{N}_{w p}^{Q}(Y, Z)$ and $S \in$ $\mathcal{N}_{w p}^{Q}(Z, X)$ such that $T=S R$. Then by assumption, there exists an $S_{0} \in \mathcal{F}(Z, X)$ such that

$$
\varepsilon \geq\left\|S-S_{0}\right\|\|R\|_{\mathcal{N}_{w p}^{Q}} \geq\left\|T-S_{0} R\right\|_{\mathcal{N}_{w p}^{Q}} .
$$

Since $S_{0} R \in \mathcal{F}(Y, X)$, we complete the proof. 
Proposition 4.10. Let $1<p<\infty$. The following statements are equivalent for a Banach space $X$.

(a) $X^{*}$ has the $\mathcal{W}_{p}-A P$.

(b) For every closed subspace $Y$ of $\ell_{p}, \mathcal{N}_{w p}^{Q}(X, Y) \subset \overline{\mathcal{F}(X, Y)}^{\|\cdot\|}$.

(c) For every Banach space $Y, \mathcal{N}_{w p}^{Q}(X, Y)=\overline{\mathcal{F}(X, Y)} \|^{\|\cdot\|_{\mathcal{N}_{p}}^{Q}}$.

Proof. (a) $\Rightarrow$ (b) and (c) $\Rightarrow$ (a) follow from Corollary 4.6.

(b) $\Rightarrow$ (c): Adapt the proof of Proposition 4.9 using Proposition 4.7.

Proposition 4.11. Let $1<p<\infty$. The following statements are equivalent for a Banach space $X$.

(a) $X^{*}$ has the $\mathcal{N}_{w p}^{Q}-A P$.

(b) For every quotient space $Y$ of $\left.\ell_{p^{*}}, \mathcal{W}_{p}(X, Y) \subset \overline{\mathcal{F}(X, Y)}\right)^{\|\cdot\|}$.

(c) For every Banach space $Y, \mathcal{W}_{p}(X, Y)=\overline{\mathcal{F}(X, Y)} \|^{\|\cdot\|_{p}}$.

Proof. (a) $\Rightarrow$ (b) and (c) $\Rightarrow$ (a) follow from Corollary 4.6.

(b) $\Rightarrow$ (c): Adapt the proof of Proposition 4.9 using Proposition 2.4.

Acknowledgement. The author would like to thank the referee for valuable comments. The author was supported by National Research Foundation of Korea (NRF2018R1D1A1B07043566).

\section{References}

[ALO] Ain, K., R. Lillemets, and E. OJA: Compact operators which are defined by $\ell_{p}$-spaces. - Quaest. Math. 35, 2012, 145-159.

[AO] Ain, K., and E. OJA: On $(p, r)$-null sequences and their relatives. - Math. Nachr. 288, 2015, 1569-1580.

[CS] Castillo, J. M. F., and F. SAnchez: Dunford-Pettis-like properties of continuous vector function spaces. - Rev. Mat. Univ. Complut. Madrid 6, 1993, 43-59.

[CK] ChOI, Y.S., and J.M. KIm: The dual space of $\left(\mathcal{L}(X, Y), \tau_{p}\right)$ and the $p$-approximation property. - J. Funct. Anal. 259, 2010, 2437-2454.

[DF] Defant, A., and K. Floret: Tensor norms and operator ideals. - Elsevier, NorthHolland, 1993.

[DOPS] Delgado, J. M., E. Oja, C. Piñeiro, and E. Serrano: The $p$-approximation property in terms of density of finite rank operators. - J. Math. Anal. Appl. 354, 2009, 159-164.

[DP] Delgado, J. M., and C. PiñeIro: An approximation property with respect to an operator ideal. - Studia Math. 214, 2013, 67-75.

[DPS1] Delgado, J. M., C. Piñeiro, and E. Serrano: Operators whose adjoints are quasi p-nuclear. - Studia Math. 197, 2010, 291-304.

[DPS2] Delgado, J. M., C. Piñeiro, and E. Serrano: Density of finite rank operators in the Banach space of p-compact operators. - J. Math. Anal. Appl. 370, 2010, 498-505.

[D] Diestel, J.: Sequences and series in Banach spaces. - Springer, New York, 1984.

[DJT] Diestel, J., H. Jarchow, and A. Tonge: Absolutely summing operators. - Cambridge Univ. Press, 1995.

[GLT] Galicer, D., S. Lassalle, and P. Turco: The ideal of $p$-compact operators: a tensor product approach. Studia Math. 211, 2012, 269-286.

[G] Grothendieck, A.: Produits tensoriels topologiques et espaces nucléaires. - Mem. Amer. Math. Soc. 16, 1955. 
[JLO] Johnson, W. B., R. Lillemets, and E. OjA: Representing completely continuous operators through weakly $\infty$-compact operators. - Bull. Lond. Math. Soc. 48, 2016, 452-456.

[K1] KIM, J. M.: Unconditionally $p$-null sequences and unconditionally $p$-compact operators. Studia Math. 224, 2014, 133-142.

[K2] KIM, J. M.: The $\mathcal{K}_{u p}$-approximation property and its duality. - J. Aust. Math. Soc. 98, $2015,364-374$.

[K3] KIm, J. M.: The ideal of unconditionally p-compact operators. - Rocky Mountain J. Math. 47, 2017, 2277-2293.

[K4] KIM, J. M.: Duality between the $\mathcal{K}_{1}$ - and the $\mathcal{K}_{u 1}$-approximation properties. - Houston J. Math. 43, 2017, 1133-1145.

[K5] KIm, J. M.: The ideal of weakly $p$-nuclear operators and its injective and surjective hulls. - J. Korean Math. Soc. 56, 2019, 225-237.

[K6] KIM, J. M.: The injective and surjective hulls of the ideal of $(p, q)$-compact operators and their approximation properties. - J. Math. Anal. Appl. 473, 2019, 71-86.

[K7] KIM, J. M.: Some results of the $\mathcal{K}_{\mathcal{A}}$-approximation property for Banach spaces. - Glasgow Math. J. 61, 2019, 545-555.

[LT1] Lassalle, S., and P. Turco: On $p$-compact mappings and the $p$-approximation properties. - J. Math. Anal. Appl. 389, 2012, 1204-1221.

[LT2] Lassalle, S., and P. Turco: The Banach ideal of $\mathcal{A}$-compact operators and related approximation properties. - J. Funct. Anal. 265, 2013, 2452-2464.

[LT3] LAssalle, S., and P. Turco: On null sequences for Banach operator ideals, trace duality and approximation properties. - Math. Nachr. 290, 2017, 2308-2321.

[LNO] Lima, A, O. NygAaRD, and E. OJA: Isometric factorization of weakly compact operators and the approximation property. - Israel J. Math. 119, 2000, 325-348.

[M] Megginson, R. E.: An introduction to Banach space theory. - Springer, New York, 1998.

[O1] OJA, E.: The strong approximation property. - J. Math. Anal. Appl. 338, 2008, 407-415.

[O2] OJA, E.: A remark on the approximation of $p$-compact operators by finite-rank operators. - J. Math. Anal. Appl. 387, 2012, 949-952.

[PP] Persson, A., and A. Pietsch: p-nukleare und p-integrale Abbildungen in Banachräumen. - Studia Math. 33, 1969, 19-62.

[P] Pietsch, A.: The ideal of $p$-compact operators and its maximal hull. - Proc. Amer. Math. Soc. 142, 2014, 519-530.

[PD] Piñeiro, C., and J. M. Delgado: p-Convergent sequences and Banach spaces in which p-compact sets are q-compact. - Proc. Amer. Math. Soc. 139, 2011, 957-967.

[R] RyAn, R. A.: Introduction to tensor products of Banach spaces. - Springer, Berlin, 2002.

[SK1] Sinha, D. P., and A. K. KARN: Compact operators whose adjoints factor through subspaces of $\ell_{p}$. - Studia Math. 150, 2002, 17-33.

[SK2] Sinha, D. P., and A. K. KARN: Compact operators which factor through subspaces of $\ell_{p}$. - Math. Nachr. 281, 2008, 412-423.

[S] Szankowski, A.: Subspaces without the approximation property. - Israel J. Math. 30, 1978, 123-129.

Received 25 March 2019 • Accepted 1 October 2019 